\title{
!F LAB: EXPERIÊNCIA DO USUÁRIO E RECURSOS NA METODOLOGIA WHAT IF IT PARA DESENVOLVIMENTO DE NARRATIVAS INTERATIVAS E IMERSIVAS ${ }^{1}$
}

\section{!F LAB: user experience and features in the "What if it" methodology for the development of interactive and immersive narratives}

\section{!F LAB: experiencia del usuario y recursos en la metodología "What if it" para el desarrollo de narrativas interactivas e inmersivas}

André Fernandes da Paz Professor Adjunto da Universidade Federal do Estado do Rio de Janeiro andredapaz@gmail.com

Sandra Gaudenzi Professora da University of Westminster S.Gaudenzi@westminster.ac.uk

\section{Resumo}

$\mathrm{O}$ artigo descreve o processo de pesquisa-ação voltado para concepção, implementação e experimentação reiterativas do programa de treinamento !F Lab e sua metodologia WHAT IF IT entre 2015 e 2018. Ambos são voltados para o desenvolvimento de projetos de narrativas digitais interativas e imersivas, orientados pela abordagem de Desing Thinking. O texto apresenta os resultados de questionários aplicados aos participantes do programa em 2017 na Europa. Também analisa os dados de entrevistas e questionários aplicados em 2018 aos participantes na Europa e aos participantes do !F Bug Lab, versão do treinamento concebida e realizada no Rio de Janeiro, Brasil.

Palavras-chave: Metodologia. Desing Thinking. Narrativa Interativa.

\begin{abstract}
The article describes the research-action process aimed at designing, implementing and experimenting with iterations to the! $\mathrm{F}$ Lab training program and its WHAT IF IT methodology between 2015 and 2018. Both are focused on the development of interactive and immersive digital narrative projects, guided by the Desing Thinking approach. The text presents the results of questionnaires applied to program participants in 2017 in Europe. It also analyses data from interviews and questionnaires to its 2018 participants and to !F Bug Lab participants, a version of the workshop conceived and held in Rio de Janeiro, Brazil.
\end{abstract}

Key words: Methodology. Design Thinking. Interactive Narrative.

\footnotetext{
${ }^{1}$ Este artigo é resultado do projeto de pesquisa "IF BUG LAB: an experimental methodology for developing interactive documentary projects in the Brazilian context", graças ao financiamento da British Academy, através do Newton Mobility Grants 2017.
} 


\section{Resumen}

El artículo describe el proceso de investigación-acción dirigido a la concepción, implementación y experimentación reiterativas del programa de entrenamiento ! F Lab y su metodología WHAT IF IT entre 2015 y 2018. Ambos se dirigen al desarrollo de proyectos de narrativas digitales interactivas e inmersivas, orientados por el enfoque de Desing Thinking. El texto presenta los resultados de los cuestionarios aplicados a los participantes del programa en 2017 en Europa. También analiza los datos de entrevistas y cuestionarios aplicados en 2018 a los participantes en Europa y los participantes del !F Bug Lab, versión del entrenamiento concebida y realizada en Río de Janeiro, Brasil.

Palabras clave: Metodología. Desing Thinking. Narrativa Interactiva.

\section{INTRODUÇÃO}

Nas últimas das décadas, a proliferação das mídias digitais e da internet abriram uma série de possibilidades narrativas, como discutidas por autores reconhecidos internacionalmente na área da comunicação (MURRAY, 1999; SCOLARI, 2013; JENKINS, 2006 e 2012). Dentro desse amplo espectro, surge novas formas narrativas interativas digitais que passam a ser chamadas por termos como documentários interativos, webdocumentários, living documentary, documentário expandido, documentário transmídia, documentário multiplataforma, conforme mapeado e analisado por uma série de autores que contribuíram para a formação de um novo campo internacional de pesquisa (ASTON; GAUDENZI, 2012; ASTON; GAUDENZI; ROSE 2017; BOLE, 2014; GAUDENZI, 2013a e 2013b; GIFREU, 2013, 2014; NASH, 2012, 2014 e 2016; PAZ; SALLES, 2013, 2015).

No recente livro publicado, Bug: narrativas interativas e imersivas (2018), André Paz e Sandra Gaudenzi, autores deste artigo, organizam a primeira introdução geral em português sobre essa produção de obras inovadoras dos últimos dez anos. Essas obras emergem com características muito diversa, com diferentes formatos, estruturas narrativas, plataformas de suporte, modos de interação, dimensões de produção: grandes produções em multiplataforma, como a referência internacional Highrise (2009-2017) ${ }^{2}$; documentários interativos, como Hollow: An interactive documentary (2012) ${ }^{3}$; obras colaborativas, como Proyecto Quipu (2014-2017) $)^{4}$; webreportagens, como Firestorm $(2013)^{5}$; narrativas mobile baseadas em aplicativos, como a ficcional Phallaina $(2016)^{6}$ ou o documentário Alma (2012) ${ }^{7}$. Em comum,

\footnotetext{
${ }^{2}$ http://highrise.nfb.ca/

${ }^{3} \mathrm{http}: / /$ hollowdocumentary.com/

${ }^{4}$ https://interactive.quipu-project.com/\#/en/quipu/intro

${ }^{5} \mathrm{https} / / / \mathrm{www}$.theguardian.com/world/interactive/2013/may/26/firestorm-bushfire-dunalley-holmes-family

${ }^{6} \mathrm{http}: / /$ phallaina.nouvelles-ecritures.francetv.fr

${ }^{7}$ http://alma.arte.tv/en/
} 
a marca de apresentar uma interface interativa e incorporar a experiência da interação como elemento fundamental da narrativa (PAZ; MACIEL, 2018).

Nos últimos cinco anos, também surgiu um grande número de narrativas imersivas digitais que se apropriam dos novos devices e plataformas de realidade virtual, vídeos em 360 e realidade aumentada, comercializados a partir do investimento massivo de grandes corporações como Facebook, Google, Sony e Samsung. Trata-se de obras como o vídeo 360 6x9: A virtual experience of solitary confinement $(2016)^{8}$, um projeto do The Guardian; a animação interativa em realidade virtual $A$ Way to $g o(2015)^{9}$; e a instalação e aplicativo em realidade aumentada de The Enemy $(2018)^{10}$. Toda essa produção inovadora, tanto de narrativas interativas como imersivas, vem sendo mapeada por exemplo pelo projeto Docubase $^{11}$, do MIT Open Documentary Lab (Massachusetts Institute of Technology), e reconhecida em festivais consagrados, como no IDFA DocLab do International Film Festival of Documentary (IDFA) de Amsterdã, nas categorais DocLab Award for Digital Storytelling e o Immersive Non-Fiction Award. Até mesmo festivais tradicionais e consagrados de cinema como Sundance, Cannes e Veneza já possuem programas específicos para essas obras em plataformas inovadoras. O campo de pesquisa apontado anteriormente incorporou as questões das narrativas imersivas, conforme fica explícito no recente livro I-Docs: The Evolving Practices of Interactive Documentary (2017) organizado por Judith Aston, Sandra Gaudenzi e Mandy Rose, também responsáveis pelo congresso I-Docs, em Bristol, Inglaterra.

Os processos criativo e produtivo dessa produção requerem uma colaboração ampla e interdisciplinar entre diversas especialidades do audiovisual, design e programação. Precisam de recursos, técnicas e conhecimentos dessas áreas, além de desenvolver novas ferramentas, processos e habilidades técnicas e estéticas. A pesquisa é uma necessidade desse ambiente de inovações. Assim, como apresentado por Paz e Salles (2015) e Paz e Jucá (2018), essa produção surgiu dentro do contexto de alguns pólos criativos na Europa e América do Norte, associada à existência de ecossistemas inovadores mais férteis que arranjam diversas iniciativas, como: os principais realizadores e financiadores de projetos, como National Film Board (NFB), RTVE, ARTE, FRANCE5, Programas Governamentais, The Fledgling Fund, Ford Foundation, MacArthur Foundation; os principais festivais e conferências (IDFA Doclab, i-Docs conference, interDocsBarcelona, Webdox, Sundance New Frontier); as

\footnotetext{
${ }^{8}$ https:/www.theguardian.com/world/ng-interactive/2016/apr/27/6x9-a-virtual-experience-of-solitary-confinement

${ }^{9}$ a-way-to-go.com

$10 \mathrm{http}: / /$ theenemyishere.org

11 https://docubase.mit.edu/
} 
plataformas e base de dados de projetos (Interdoc, MIT Docubase, DocShift Summit), produções da grande mídia (Le Monde, The New York Times, The Guardian); empresas produtoras inovadoras (Upian, Honkytonk Films, Submarine Channel, Helios Design Labs), eventos e mercados catalisadores de novos projetos (Cross Video Days, Sunny Side of the Doc, Sheffield DocFest).

Em todo esse cenário, a produção não apresenta um formato hegemônico. Há alguns um pouco mais consolidados, mas a grande marca é a presença de obras híbridas e um processo de constante inovação. Além disso, como coloca Paz e Maciel (2018), a interface é apenas um núcleo de cruzamentos de práticas de interação, agenciamento e engajamento. Em consonância com a perspectiva da estética relacional, conforme vem sendo desenvolvida por Paz (PAZ; SALLES, 2013 e 2015; PAZ, 2017), mais do que uma obra enquanto produto, trata-se de um processo de práticas interativas e relacionais entre equipe, participantes e interatores, para o qual não há um modelo a seguir. Em grande parte dessas obras, os processos criativo e produtivo consistem em, portanto, antes de se produzir conteúdo, conceber um dispositivo próprio, mais amplo, muito além da interface e do aspecto tecnológico e formal. Ou seja, é preciso realizar um cruzamento específico de práticas e processos relacionais em consonância com o formato e sua interface.

Todo esse quadro acentua ainda mais a relevância de uma série de hackathon, labs, treinamentos já existentes nos ecossistemas apontados para apoiar o desenvolvimento de novos projetos. São iniciativas que trazem abordagens, metodologias e ferramentas de storytelling, desing thinking, desenvolvimento de software. No contexto de uma verdadeira cultura hackathon $^{12}$, surgiu, por exemplo, o Interactive Documentary Workshop (ID W) ${ }^{13}$, o Hackastory Workshop ${ }^{14}$ e novas iniciativas em universidades ${ }^{15}$. Assim como outros, o já comentado IDFA criou o IDFA DocLab Academy, para apoiar também a elaboração de projetos seguida de sessões de pitching. Todas essas iniciativas evidenciam a necessidade dos criadores e produtores adotarem uma nova práxis de produção. Em paralelo, mais recentemente, os pesquisadores têm se debruçado sobre as metodologias adotadas nessas iniciativas. O já comentado Aston, Gaudenzi e Rose (2017) tem toda uma seção dedicada aos novos métodos, com artigos que mapeiam não apenas as metodologias apontadas como

\footnotetext{
12 Ver alguns exemplos: POV Hackthons (http://www.pbs.org/pov/hackathon), Tribeca Hacks (https://www.tfiny.org/pages/tribeca_hacks_about), Popathons (http://popathon.org).

${ }^{13}$ Ver http://idw.supsi.ch/

${ }^{14}$ Ver https://hackastory.com

15 Ver como exemplo o Digital Storytelling Lab da Columbia, (http://www.digitalstorytellingLab.com), o Digital and Storytelling Lab da University of Westminster (http://www.digitalstorytellingLab.com) e o Transmedia Zone na Ryerson's University (http://transmediazone.ca).
} 
aquelas usadas internamente pelas próprias produtoras em seus projetos (LININGTON, 2017; PRINGLE, 2017; GAUDENZI; WEILER, 2017).

No mesmo livro, em "User experience versus author experience: lesson learned from the UX Series", Gaudenzi (2017) aponta como começou uma pesquisa-ação ao criar em 2015 o programa de treinamento !F LAB, que é objeto de estudo deste artigo ${ }^{16}$. Em 2014, a autora havia realizado uma pesquisa chamada $U X$ Series. Nesta, entrevistou uma série de produtores de $i$-docs sobre seus métodos, descobrindo o quão pouco aplicavam princípios e práticas de user centered design e desenvolvimento de softwares. Enquanto esses últimos estão focados em identificar a audiência e encontrar soluções adaptadas a sua experiência, os produtores e diretores vindo do audiovisual estão focados em um processo criativo como uma espécie de expressão de seu self. Essa tensão entre user experience e author experience precisava ser encaminhada nos projetos de narrativas interativas e imersivas. Nesse sentido que Gaudenzi então criou o IF LAB como um programa de treinamento que adota princípios de storytelling e métodos ágeis, orientado pela abordagem de Desing Thinking, enquanto aplica e desenvolve sua própria metodologia - o WHAT IF IT process. De 2015 a 2018, o programa foi realizado pelo IDrops ${ }^{17}$ em diferentes lugares da Europa e financiado pela UE, em paralelo a uma pesquisa voltada para experimentar melhorias correlacionadas e forma cíclica. Este artigo descreve com mais detalhes a proposta do !F LAB, a metodologia WHAT IF IT e as impressões dos participantes - os usuário da metodologia. No item 2. 3, o artigo aponta os resultados de questionários aplicados aos participantes de 2017 em dois momentos distintos, em particular sobre as pressuposições e efeitos da metodologia WHAT IF em relação à mudança de mentalidade (mind shift) e a tensão entre user experience e author experience inerente ao processo.

Os resultados da pesquisa sobre o programa de 2017 foram satisfatórios, mas surgiu outra questão em paralelo a ser investigada. Era evidente para Gaudenzi que os participantes passavam grande parte de seu tempo lutando por fundos e equipe para produzir seus projetos. Isso colocava em questão a capacidade dos projetos serem realizados posteriormente ao programa e, portanto, a pertinência da metodologia, sobretudo, para circunstâncias onde os recursos eram mais limitados. Por isso surgiu o projeto de pesquisa e colaboração internacional dos autores deste artigo, dedicado a entender um pouco melhor a relação entre

\footnotetext{
${ }^{16}$ A pesquisa-ação consiste, sumariamente, na articulação entre a pesquisa teórica e uma prática de intervenção. Os pesquisadores são co-realizadores do fenômeno estudado (Desroche, 2006).

${ }^{17}$ http://idrops.org/en/
} 
os recursos disponíveis e o processo de desenvolvimento de projetos. Graças ao apoio da British Academy, através do Newton Mobility Grant, ambos iniciaram uma pesquisa-ação voltada para o desenvolvimento da metodologia. Esse projeto se chamou !F Bug Lab e consistia na concepção, realização e pesquisa de uma versão adaptada do !F Lab ao contexto brasileiro.

Como apresentam Paz e Salles (2015) e Paz e Jucá (2018), a produção brasileira não havia acompanhado nos dez anos anteriores o volume e a sofisticação dos pólos criativos internacionais, justamente por não contar com ecossistemas inovadores, o que limita bastante os recursos financeiros e técnicos disponíveis. Em 2015, o Bug $404^{18}$ havia sido concebido por André Paz como uma rede e portal de informação na internet que buscasse contribuir nesse sentido, ao estimular o diálogo e colaboração entre pesquisadores de diversas áreas e produtores, artistas, gestores, interessados em narrativas interativas e imersivas no Brasil. Desde então a iniciativa realizou uma série de ações, como oficinas, encontros, publicações. Em 2018, o bug404 foi responsável pelo projeto da Mostra Bug, a primeira e maior mostra do campo já realizada no Brasil. Durante o evento, André Paz coordenou o Buglab: conferência internacional. Foi neste contexto que foi realizado em setembro de 2018 o treinamento !F Bug Lab por quatro dias, concebido e liderado por Paz e Gaudenzi.

O !F Bug Lab foi mais uma rodada de reiteração da pesquisa-ação de desenvolvimento da metodologia WHAT IF IT. O item 2.4 deste artigo apresenta as questões de pesquisa que orientaram a concepção do workshop no Brasil. Conforme explicado a seguir, foi elaborada uma atividade especifica baseada nos princípios da Effectuation, teoria construída a partir das contribuições de Saras Sarasvhaty (2001, 2003, 2005) e bastante consolidadas no campo do estudo do empreendedorismo. O item 2.5 apresenta a análise dos dados então levantados pelas entrevistas no !F Lab na Europa e no !F Bug Lab no Rio de Janeiro. Os resultados permitem avaliar as próprias pressuposições da concepção do workshop e suas atividades e feitos a partir das impressões e avaliações dos participantes, os verdadeiros usuários da metodologia.

\section{DESENVOLVIMENTO}

\section{1 !FLAB}

${ }^{18}$ Bug404.net 
!F Lab (Interactive Factual Lab) é um programa de treinamento em forma de workshop concebido e liderado por Sandra Gaudenzi, produzido pela agência belga de inovação social !DROPS e financiado pela União Européia. Os workshops aconteceram cada ano em diferentes localidades da Europa, visando apoiar "non-fiction storytellers" para idealizar e desenvolver suas narrativas interativas e imersivas usando plataformas digitais como a Internet, dispositivos móveis, óculos de Realidade Virtual ou Realidade Aumentada ou ambientes imersivos. Entre 2015 e 2018, !F Lab atendeu 72 projetos participantes de 25 países e criou uma verdadeira comunidade de !Flabbers que contiua ativa e em contato via mídias sociais. !F Lab parou suas atividades em 2019, seguindo a mudança na política de financiamento da Creative Europe.

A idéia original era ser uma mini incubadora na Europa onde narrativas interativas e imersivas poderiam ser testadas, mentoradas e desenvolvidas. Gaudenzi havia notado que a produção até então existente se dividia entre: por um lado, em experimentações de formato pelos grande mainstream grupos e agências, que detinham recursos, em especial equipe treinada; do outro, produtores independentes e filmmakers que não detinham recursos financeiro se técnicos para tal. Era oportuno, no começo de 2015, apresentar à Creative Europe um programa de treinamento para o desenvolvimento de projetos, onde novos entrantes poderiam ser guiados e mentorados, enquanto uma metodologia poderia ser testada e divulgada para um público amplo.

A primeira edição do !F Lab, em 2015, foi baseada na idéia de um programa dividido em três workshops de quatro dias cada, em um período de seis meses: Story Booster, direcionado para se definir o principal propósito do projeto e a plataforma mais adequada, baseado em uma abordagem de User Centered Design; Design Booster, focado na consolidação de um primeiro protótipo em papel (paper prototype) com a respectiva testagem; e o Prototype Booster, voltado para realização de um primeiro protótipo digital, com a ajuda de programadores externos. Em média, oito a doze projetos foram selecionados depois da avaliação de suas candidaturas. Um grupo principal de três profissionais (um designer, um produtor de TV e um creative technologist) fixos foram agrupados como coaches para acompanhar os três boosters. Gaudenzi participava como um quarto coach, com sua experiência no campo das narrativas interativas e imersivas, responsável pelo processo de coordenação e facilitação. Em cada Booster, um grupo extra de especialistas ad hoc for am convidados para ajudar os participantes em tarefas específicas de design, desenvolvimento conceitual e planejamento financeiro. 
A primeira configuração do !F Lab em 2015 foi concebida por um processo colaborativo de brainstorming e pesquisas pessoais de Gaudenzi com a equipe de coaches. No final do primeiro ano, todos avaliaram os resultados e iniciaram a consolidação de uma metodologia. Em 2016, o !F Lab foi importante para testar e adaptar as atividades da metodologia proposta e mapear os momentos chave de desenvolvimento interativa, mesclando com princípios de User Centered Design, metodologias ágeis e conhecimento e práticas de storytelling. O resultado desse processo é que foi chamado de WHAT IF IT, uma metodologia para concepção e desenvolvimento de projetos interativos e imersivos, lançada no !F Lab 2017. A esta altura, foi também decidido diminuir os Boosters para dois workshops de cinco dias (Story Booster e Prototype Booster), uma vez que os participantes tinham pouco tempo e recursos para trabalhar entre os boosters, quando voltavam para suas casas e países de origem. Era mais proveitoso mantê-los concentrados em dois workshops mais longos com dois meses de intervalo. Por outro lado, a produção do !F Lab concentrou mais recursos no Prototype Booster onde os participantes passaram a contar com três dias de ajuda direta de tecnólogos e designer consultores externos, com um ritmo e produtividade de um verdadeiro hackathon. Já para 2018, !F Lab desenvolveu uma série de cards e canvas que serviram como um passo a passo estruturante do processo WHAT IF IT, como resultado dos quatro anos anteriores de atividades e pesquisa-ação. O item seguir descreve essa versão do WHAT IF IT.

\subsection{A metodologia WHAT IF IT}

Figura 1. Slide de apresentação do processo WHAT IF IT

\section{Mixing methodologies}

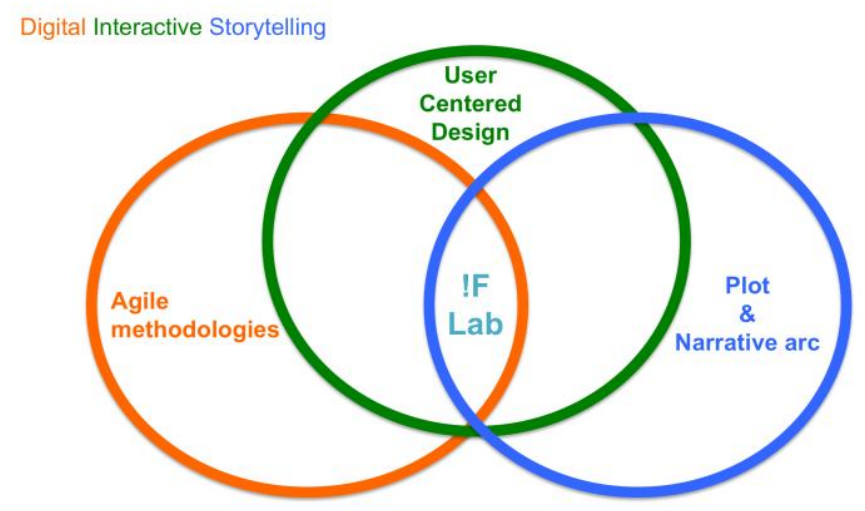

Fonte: Cortesia !F Lab 
Pensado como uma metodologia na interseção de três diferentes áreas, como ilustra a figura 1 acima, o processo WHAT IF IT começa perguntando o que (WHAT) é o principal conceito do projeto? A partir de então questiona a anteratividade (I), formula (F) os impactos desejados, para idealizar (I) o protótipo e finalmente testá-lo (T), antes de começar o ciclo de novo. É um processo que utiliza design thinking para ajudar os criadores e produtores a encontrarem a melhor plataforma e estratégia de comunicação interativa para alcançar seus objetivos, em suas narrativas, de maneira que possam ser escutados pela sua target audience. Nesse sentido, o processo consiste na repetição reiterativa das cinco fases ilustradas na figura 2 abaixo.

Figura 2. As cinco fases do processo WHAT !F IT

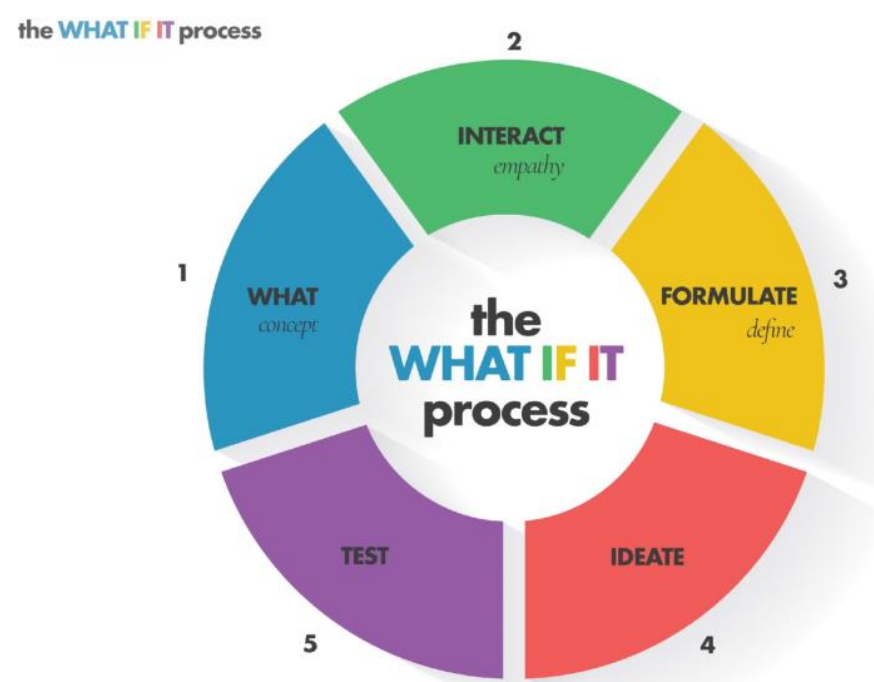

Fonte: iflab.net

Quando se utiliza uma abordagem de User Centred Design em um contexto de storytelling, o desafío é fazer acreditar que ao se começar pelas necessidades do usuário - ao invés do desejo autoral de se contar a história de determinada forma -, o conceito da forma de interatividade a ser adotada emerge naturalmente por si mesmo. Essa abordagem de pensamento criativo é totalmente estranha para alguém que foi treinado como um jornalista, diretor de cinema ou documentarista, de maneira que é preciso experimentar para acreditar.

Para guiar os participantes pela exploração de possibilidades de plataformas interativas e a mudança de mentalidade autoral, foram criados dois tipos de ferramentas: Cards e Canvases. Os cards são mini lições oferecidas pelos coaches, enquanto os canvases são 
exercícios práticos concebidos para ajudar as equipes a tomarem decisões para seus projetos antes de se moverem para os passos seguintes. A alternância entre mini lições e exercícios práticos foi desenhada para inspirar e focar os participantes em seus projetos, que se movem constantemente entre teoria e prática. A figura 3 abaixo apresenta a sequência e temáticas de cards e canvases organizada pelas fases da metodologia conforma apresentado anteriormente de forma circular.

Figura 3 - Cards e Canvases do WHAT !F IT em 2018

\section{CARDS \& CANVASES OVERVIEW IF CARD}

\begin{tabular}{|c|c|c|c|c|}
\hline WHAT CONCEPT & INTERACT & FORMULAIIE & IDEATE & TEST \\
\hline 吕 & 吕.1 & 吕 3.1 & 吕 4.1 & 占 5.1 \\
\hline What: Story \& Purpose & Users research & Project Challenge & $\begin{array}{l}\text { User Journeys } \\
\text { (from text to sketches) }\end{array}$ & Paper Prototype \\
\hline ص1.2 & 吕 2.2 & E 3.1 & 占 4.2 & מ 5.2 \\
\hline WHY: Impact & User Modeling & User's Impact Canvas & $\begin{array}{l}\text { HOW: Wireframes \& } \\
\text { Interactive Storyboards }\end{array}$ & UX Evaluation \\
\hline ص1.3 & $\begin{array}{l}\text { E } 2.1 \\
\text { Personas Canvas }\end{array}$ & 气 3.2 & & \\
\hline $\begin{array}{l}\text { WHO: Target } \\
\text { Audience }\end{array}$ & E 2.1 & Levels of Impact Canvas & & \\
\hline $\begin{array}{l}\text { El.1 } \\
\text { Concept Canvas }\end{array}$ & $\begin{array}{l}\text { Semi leading Interviews } \\
\text { Empathy Map }\end{array}$ & $\begin{array}{l}\text { - } 3.3 \\
\text { Project Challenge } \\
\text { Canvas }\end{array}$ & & \\
\hline
\end{tabular}

the WHAT IF IT process

Fonte: iflab.net

No primeiro dia do Story Booster, os participantes são convidados a preencherem o Concept Canvas (Figura 4 abaixo), colocando ênfase nas questões que destacam: qual (WHAT) é sua história (storytelling)?; quem (WHO) é sua audiência? (user centred design); porquê (WHY) deveria ser interativo? (technology). A quarta parte do mapa conceitual tem a ver com os impactos desejados: porquê (WHY) seu projeto é relevante para você (participante) e o que você deseja que sua audiência saiba/sinta/faça depois de experimentar seu projeto? A questão central para essa metodologia é o conceito de coerência: história, design, audiência e impacto precisam estar alinhados e relacionados mutuamente. 
Figura 4 - Exemplo de Canvas: The Concept Canvas

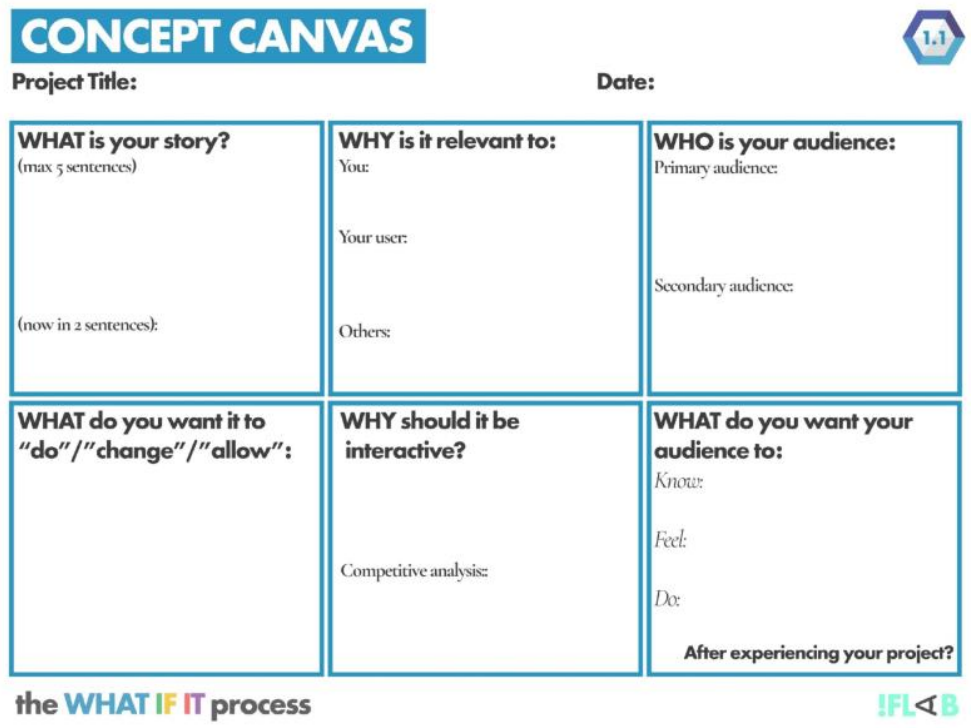

Fonte: iflab.net

Os cinco dias que se seguem do Story Booster são dedicados a aprofundar o questionamento de cada um desses pontos de decisão de uma forma reiterativa através de ferramentas como construir uma Persona e fazer um user-empathy map, definindo os desafios do projeto, desenhando a user journey e wireframes iniciais, construindo o paper prototype e testando-o no contexto do !F Lab. O Story Booster termina com um pitch informal entre os participantes dos próprios projetos e coaches, com o objetivo de assimilar feedbacks a serem processados quando repetirem todo o ciclo novamente em suas casas e locais de origem, mas agora com representantes de suas audiências reais.

Dois meses depois, todos se reúnem novamente para o Prototype Booster, prontos para transformar o paper prototype em digital prototype: a primeira representação visual interativa de seus projetos. Durante esses dias de hackathon os participantes se dão conta de como a colaboração com designers e creative technologists é essencial nesse campo tão multidisciplinar. O produto final desses dias é apresentado para uma banca de profissionais do mercado, sejam financiadores ou produtores. Esse último pitch, agora formal, tem a mesma função do anterior do Story Booster: mais uma oportunidade reiterativa dos participantes incorporarem os feedbacks da banca para aprimorarem suas demos, agora já mais 
desenvolvidas para serem apresentadas a potencial financiadores que pudessem levar o projeto a verdadeira produção.

\subsection{Avaliação do WHAT IF IT 2017}

Ao final de cada !F Lab Booster, os participantes respondem um formulário de avaliação da metodologia. No ano de 2017, em especial foi feita uma pesquisa mais dedicada através da aplicação de questionários anônimos em dois momentos. O primeiro foi respondido logo após terminarem o Prototype Booster, quando a experiência ainda estava recente, e o segundo foi aplicado oito meses após o fim do treinamento. O intervalo foi proposital no sentido de tentar entender melhor que práticas e ferramentas ainda estavam sendo utilizadas pelos participantes. Seis dos oito projetos que participaram dos boosters responderam ambos questionários. A idade das pessoas que responderam variou de 21 a 49 anos e todos eles vinham de um background da área do storytelling (quatro deles eram documentaristas, um predominantemente fotógrafo e um pesquisador de cultura visual).

O primeiro questionário foi aplicado por formulário do Google com nove questões: quatro de múltiplas escolhas, quatro semiabertas e uma aberta. As perguntas semiabertas foram orientadas para refletir a impressão dos participantes e expressar opiniões negativas ou positivas. Por exemplo: "O fato de haver uma metodologia passo a passo lhe ajudou a desenvolver sua história interativa? Explique de que forma." Outras perguntas foram mais abertas, como: "O fato de começar o processo criativo pela perspectiva da audiência e impacto foi intuitivo para você? Por favor explique." A intenção era refletir mais sobre a própria metodologia. As questões de múltipla escolha se voltaram para entender o impacto do processo em seus projetos, como: "No !F Lab nós lhe pedimos para se concentrar na experiência do usuário (e dar um pouco menos de importância à história). Isso foi...: 1 . Frustrante para você como autor, mas necessário; 2. Frustrante e desnecessário - você não faria isso outra vez; 3. Nada frustrante, na verdade um alívio (muitas decisões foram resolvidas pelo teste); 4. Eu tive que mudar completamente minha maneira de criar então isso foi frustrante no começo, mas muito necessário para abrir a perspectiva". Os resultados desta última pergunta, em particular, encontram se no gráfico 1 abaixo. 
Gráfico 1 - Questão n.6

6. At IF Lab we asked you to concentrate on the user experience (and gave slightly less importance to the story itself). Was that...

6 responses

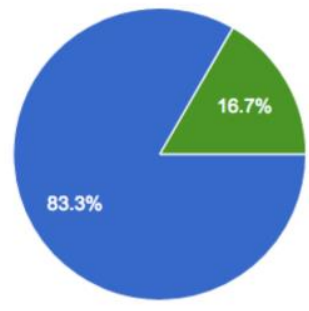

$$
\begin{aligned}
& \text { frustrating to you as an author, but } \\
& \text { necessary } \\
& \text { frustrating and not necessary - you } \\
& \text { will not do it next time } \\
& \text { not frustrating at all, actually quite a } \\
& \text { relief (as a lot of decisions are solve } \\
& \text { themselves via testing) } \\
& \text { I had to shift completelly my way of } \\
& \text { creating so it was frustrating first, but } \\
& \text { very necessary to open the perspec... }
\end{aligned}
$$

Fonte: !F Lab

Os resultados da pesquisa são apresentados em detalhes em "The WHAT IF IT Process, Moving from Story-telling to Story-experiencing" (Gaudenzi, 2019), mas as principais considerações podem ser resumidas da seguinte forma:

1. O principal mindshift notado pelos participantes foi que seguir a medotodologia de User Centered Design os forçava os a colocar o usuário no centro do processo criativo. Isso era algumas vezes um processo desconfortável (como mostra o gráfico 1 acima), mas eles notaram o quanto isso tornou evidente as próprias pressuposições e tornou os projetos mais claros.

2. O processo WHAT IF IT desafiou a percepção dos participantes sobre o próprio papel como autores. Sair um pouco da responsabilidade de criar histórias com personagens para desenhar uma experiência do usuário ouvindo os usuários finais mudou o equilíbrio da autoria entre autor, creative technologist e usuário. Essa mudança não foi fácil de ser ajustada.

3. Oito meses depois do final do !F Lab, todos os participantes que responderam estavam ainda trabalhando em seus projetos e a maioria (66\%) declararam estar usando parte da metodologia WHAT IF IT, conforme apresenta o gráfico 2 abaixo. 
Gráfico 2 - Questão n.2

\section{If yes, did you re-use the WHAT IF IT methodology?}

6 responses
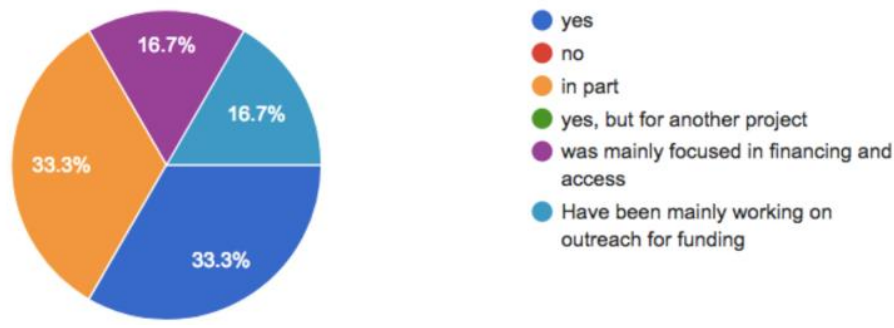

Fonte: !F Lab

As impressões dos participantes sobre o mind shift no qual se baseia a metodologia indicam uma avaliação positiva da experiência e possíveis efeitos a longo prazo, o que reforçava a necessidade de seguir os princípios fundamentais de User Centre Design. Por outro lado, como dito anteriormente, independente dos questionários, a importância dos recursos de cada equipe para realizar seus projetos não parecia ter a atenção devida, de acordo com as observações de Sandra Gaudenzi. Pressionar os participantes para pensarem grande e fora da caixa tinha seus limites se eles não tivessem chances para realizar tal projeto. Foi nesse sentido que surge o projeto de pesquisa-ação !F Bug Lab para ser realizado em 2018.

\section{4 !F Bug Lab: projeto de pesquisa}

No início deste artigo, algumas características dos processos de criação e produção de narrativas interativas e imersivas foram apontadas: ausência de formato, obras híbridas, multidisciplinaridade, necessidade de ecossistemas inovadores. Toda essa necessidade de inovação traz riscos para os projetos. É justamente por isso que eles precisam estar mais do que nunca amparados nos recursos disponíveis para sua realização. Nesse sentido, André Paz e Sandra Gaudenzi foram buscar princípios de orientação para algumas práticas do programa !F Lab na teoria conhecida como Effectuation, construída a partir das contribuições de Saras Sarasvhaty e bastante consolidadas no campo do estudo do empreendedorismo. 
De uma maneira bem simples, Effectuation é o resultado de uma pesquisa sobre como os empreendedores tomam decisão e agem em ambiente inovadores de alto risco. Saras Sarasvhaty (2001, 2003, 2005), uma psicóloga cognitiva, conduziu uma pesquisa com empreendedores de sucesso para entender como eles se comportam diante do desenvolvimento de novos produtos. Nesses ecossistemas de alto risco, ela identificou uma lógica de pensamento específica, a que chamou de effectual logic. Em termos gerais, eles projetam os fins possíveis com os meios disponíveis. É a partir dos recursos disponíveis entendidos em um sentido amplo, como financeiros, habilidades, parcerias - que eles concebem, desenvolvem e produzem seus produtos ou serviços. Isso acontece dentro do que Sarasvhaty chama The Effectual Cycle: a expansão cíclica dos recursos em paralelo a redefinição dos produtos e objetivos.

Como o processo de pesquisa-ação !F Lab poderia incorporar esses princípios da Effectuation na metodologia WHAT IF IT para melhor encaminhar o papel dos recursos no processo criativo? Como adaptar essas proposições que dizem respeito a um processo empreendedor mais longo a um processo mais curto de desenvolvimento de projeto? Essas perguntas não poderiam ser respondidas de forma exclusivamente teórica. $\mathrm{O}$ projeto de pesquisa-ação !F Bug Lab se propôs a conceber e experimentar uma versão adaptada do !IF Lab que levasse em consideração esses princípios. A pesquisa começou, entretanto, com a participação de André Paz como coach e pesquisador nos Story Booster e Prototype Booster do !F Lab em 2018 realizados em Riga (Letônia) em maio e Ghent (Bélgica) em julho. Nesses, foi realizada uma entrevista presencial com perguntas semi-abertas e abertas com todos os participantes, que estavam focadas em entender a história do projeto, os recursos disponíveis desde o início de sua concepção e o perfil dos produtores. As perguntas buscavam identificar a origem do projeto e mapear a relação de seu desenvolvimento e produção com os recursos disponíveis para cada equipe.

Durante os encontros, Paz e Gaudenzi elaboraram a proposta do treinamento !F Bug Lab que viria a ser realizado no final de setembro no Rio de Janeiro, Brasil. Por restrições de recursos da própria produção do treinamento e disponibilidade dos participantes, tudo viria a ser realizado concentrado em um workshop de 4 dias inteiros, como um Story Booster. Diferente, entretanto, das outras versões, o workshop se iniciaria com uma dinâmica baseada no que ficou chamado de Strategy Canvas, que, após uma curta apresentação da Effectuation, convidada os participantes a fazerem uma reflexão sistemática sobre os propósitos e características gerais do projeto e os recursos disponíveis, para a fase de desenvolvimento do 
projeto e para fase posterior de produção: equipe, equipamentos, parcerias, financiamento. $\mathrm{Na}$ oficina, foi reforçada a necessidade desses recursos mapeados estarem em sintonia com a proposta geral do projeto. Assim, o workshop se dividia duas fases seguidas de forma cíclica (Strategy canvas e paper prototype), no intuito de reforçar a coerência entre recursos e o produto desenvolvido, seguidas de um pitch final para uma banca de avaliadores externos do mercado. Durante as duas primeiras fases também foram implementadas mais rodadas de apresentação e feedbacks entre pares de equipes de projetos participante, seguindo indicações das avaliações dos participantes nos boosters da Europa.

Assim como nos boosters do !F Lab 2018a, no final do !F Bug Lab foi feita uma entrevista presencial com todos os participantes voltada para identificar a origem do projeto e mapear a relação de seu desenvolvimento e produção com os recursos disponíveis para cada equipe. Os participantes no Brasil, assim como na Europa, como esperado, se mostraram muito abertos a falarem de seus projetos. No entanto, era preciso uma pesquisa sobre a avaliação deles sobre as atividades do programa, em especial as diferentes fases e a inovação implementada de se pensar o produto a partir dos recursos: a relação das fases paper prototype e strategy canvas. Para isso foi aplicado um questionário anônimo através de formulário do google com 24 perguntas, da quais 19 se tratavam de perguntas em Escala de Likert ( 0 a 5) sobre a avaliação dos participantes sobre as diversas fases, atividades, coaches $e$ consultores do projeto. As outras questões eram semi-abertas e abertas sobre as principais contribuições do workshop e possíveis sugestões. Esses formulários foram aplicados na semana posterior ao final do evento, de maneira que as lembranças ainda estavam frescas e os participantes não se sentiriam constrangidos e darem respostas enviesadas. Os resultados das entrevistas e questionários é explorado no item a seguir.

\subsection{IF Bug Lab: Resultados}

O !F Lab 2018 teve doze projetos selecionados com um a três integrantes participantes de cada equipe, de sete países da Europa. Quatro dessas equipes (33\%) eram originárias do setor audiovisual, sendo uma delas da fotografia - o que mostra uma diversificação em relação aos anos anteriores, onde a presença de equipes oriundas do audiovisual era maior. Dois desses projetos (16\%) vinham diretamente de pesquisas ou projetos de dentro das universidades. Já o !F Bug Lab contou com sete projetos participantes com dois ou três integrantes por equipe de três estados diferentes do Brasil (e deles do eixo RJ-SP). 
Praticamente todas as pessoas das diferentes equipes atuavam no setor audiovisual, seja com televisão, cinema, novas mídias ou pesquisa. Quatro projetos (57\%) eram de pessoas que atuavam na universidade, com ensino, pesquisa ou divulgação científica.

Esse quadro mostra uma diferença clara: os participantes no Brasil se assemelhavam ao perfil das equipes dos primeiros !F Labs, praticamente do setor audiovisual e universidades. Nesse sentido, é preciso destacar que, em ambos os casos, os participantes foram selecionados a partir de uma série de inscritos que souberam do treinamento a partir de divulgações bem distintas. Na Europa, o !F Lab já existia há quatro anos e contava com uma rede de divulgação via i-docs, iDrops e os próprios iflabbers, com um alcance mais amplo. No Brasil, a divulgação ficou bastante concentrada via mídias sociais do bug404, o que claramente influenciou um universo de inscritos de produtores pequenos e independentes, advindos em grande parte do audiovisual e universidade.

No !F Lab 2018, apenas um projeto teve pequenas modificações ao longo dos workshops, os outros todos tiveram modificações bem mais consistentes nas idéias originais. Esse também foi o padrão encontrado no Brasil, onde apenas um projeto já estava mais desenvolvido. Mas quando perguntados sobre qual eram as razões pelas quais o projeto não havia ainda sido realizado e o porquê da opção pelo treinamento, os participantes da Europa apontaram principalmente razões e necessidades relacionadas ao desenvolvimento de seus projetos. Já os brasileiros destacaram esse ponto, mas também a necessidade de se buscar financiamento e o interesse em aprender a própria metodologia - associado ao reconhecimento dos nomes e trabalhos dos facilitadores e coaches.

Quanto aos recursos (financiamento, habilidades, equipamentos e parcerias), a entrevista dividiu as perguntas sobre a disponibilidade e necessidade dos mesmos em duas fases: para o desenvolvimento do projeto - durante as oficinas e os intervalos entre os boosters - e para a produção futura realmente do projeto. No caso do !F Lab 2018, para o desenvolvimento do projeto, cinco projetos já tinham algum tipo de financiamento para o transporte e presença da equipe nos boosters, além de algum apoio financeiro para pequenos curtos extras. Os outros estavam arcando as despesas com seus próprios recursos financeiros. Quatro projetos destacaram a necessidade de habilidades técnicas (Skills) que não possuíam, em particular conhecimentos de programação ou design, que em parte acabaram sendo complementadas no Prototype Booster. Já no caso brasileiro, dos sete projetos, cinco destacaram a necessidade de conhecimento e habilidades técnicas, sobretudo programação, uma vez que vinham do audiovisual e planejavam produtos de documentário interativo, 
docugame ou aplicativo. Apenas dois projetos já tinham alguma forma de apoio financeiro para participar das oficinas. Esse quadro mostra a importância do apoio de consultores e técnicos de desing e programação, ainda mais para um processo criativo que depende da colaboração das diversas especialidades desde a etapa original de concepção. O !F Bug Lab não contou uma fase de digital prototype, mas teve dinâmicas de consultoria técnica de voluntários especializados, entre outras coisas, em programação e design. Esses resultados evidenciam a importância desse apoio, mas sinalizam também a necessidade de um reforço dessas atividades em outros treinamentos futuros.

Quanto aos recurso avaliados para a possível futura produção dos projetos, é importante destacar que no !F Lab 2018, quatro projetos já tinha financiamento para essa fase posterior ao treinamento. Enquanto no !IF Bug Lab, nenhum tinha esse financiamento garantido e apenas duas equipes tinha noção mais clara de onde poderiam levantar financiamento. No entanto, todos os projetos destacavam as parcerias já existentes como um recurso claro em suas mãos para a fase de produção. Resumindo, as entrevistas mostravam que o grupo do !F Bug Lab vinha do setor audiovisual, necessitava de apoio técnico (Programação e design) para conceber e desenvolver suas propostas, não tinha qualquer financiamento para a produção de seus projetos, apontava suas parcerias como um recurso disponível e oportuno central para futura produção - confirmando um dos pressupostos do !F Bug Lab: de que trabalharia com participantes com recursos mais limitados. Uma vez melhor caracterizado o perfil dos participantes, os resultados dos questionários podem ser melhor compreendidos.

O questionário anônimo através de formulário do google tinha 19 perguntas em Escala de Likert (0 a 5), das quais destacamos aqui as que diziam respeito à avaliação do Strategy Canvas, criado para o !F Bug Lab, e do Paper Prototype. Doze participantes das diferentes equipes responderam ao questionário. Quando perguntados sobre a contribuição do Strategy Canvas para o desenvolvimento do projeto durante a oficina, os resultados foram bastante satisfatórios. Conforme o gráfico 3 abaixo, dez pessoas (83\%) apontaram avaliação positiva (4 ou 5) e sete (58\%) marcaram avaliação máxima (5). Quando a pergunta dizia respeito à influência em projetos futuros, após o treinamento, esses números passaram para 11 (92\%) pessoas com avaliação positiva (4 ou 5). 
Gráfico 3 - Avaliação da contribuição do Strategy Canvas

De 1 a 5, como você avalia a contribuição da fase Strategy Canvas (Pensar a Proposta, Referências, Recursos, Financiamento) para o desenvolvimento de seu projeto específico durante a oficina?

12 responses

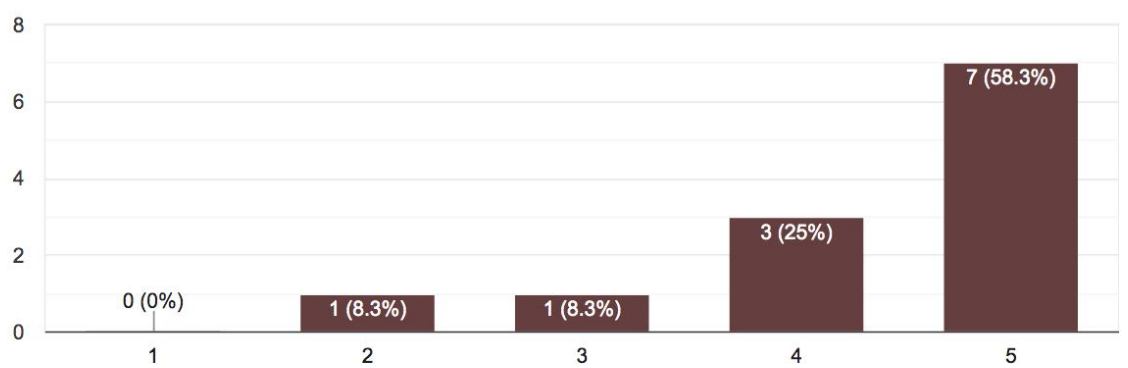

Fonte: Autores

A avaliação da influência da fase Paper Prototype no desenvolvimento do projeto durante a oficina foi ainda mais satisfatória - conforme esperado, pois já vem sendo testada há quatro anos na Europa e consiste na base principal da metodologia aplicada. Todas as pessoas (100\%) deram uma avaliação positiva (4 ou 5), sendo que 10 (83\%) apontaram avaliação máxima. Ao avaliar a contribuição dessa fase para aprimorar a forma de desenvolver projetos futuros, $11(92 \%)$ pessoas atribuíram nota máxima (5).

Como já explicado, o Strategy Canvas foi inserido para que reforçar a reflexão sobre os recursos, dentro da proposta e estratégia geral do projeto, e a sintonia entre esses e o protótipo concebido. Nesse sentido, como aponta o gráfico 5, quando perguntadas como avaliavam a influência do Canvas sobre as decisões do Paper Prototype, 11 (92\%) das pessoas apontaram positiva (4 ou 5) e $7(58 \%)$ deram avaliação máxima. O inverso também foi avaliado em outra pergunta, todos (100\%) consideraram positiva (4 ou 5) e $6(50 \%)$ deram nota máxima para a influência do Paper Prototype no Strategy Canvas.. Esses resultados evidenciam a influência cíclica entre ambos, conforme o objetivo para o qual o Strategy Canvas foi criado. 
Gráfico 4 - Influência do Strategy Canvas no Paper Prototype

Como você avalia a influencia da fase Strategy Canvas (Pensar a Proposta, Referências, Recursos, Financiamento) nas decisões da fase Paper Prototype (Audiência, Persona, Impacto, User Flow, User Journey)?

12 responses

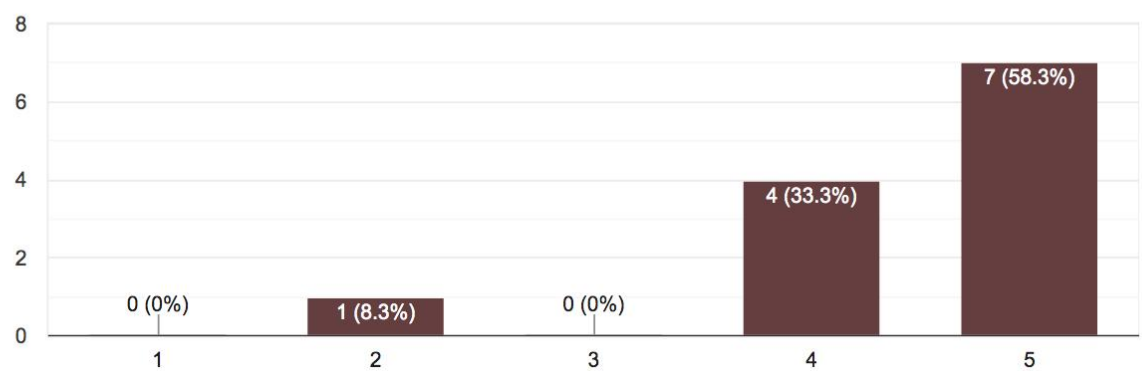

Fonte: Autores

A avaliação do incremento de dinâmicas de trocas diretas entre os participantes, outra modificação em relação ao !F Lab, foi bastante positiva: 10 pessoas $(83 \%)$ deram avaliação máxima (5) e 2 (17\%), nota 4. Quando perguntados "o quanto a oficina contribuiu para o desenvolvimento do seu projeto?", dez pessoas (83\%) responderam com a nota máxima (5) e todas $(100 \%)$ avaliaram positivamente (4 ou 5$)$. Sete pessoas $(58 \%)$ consideraram ainda que projeto apresentado no pitch final era "substancialmente diferente do projeto inicial apresentado para a oficina". Todos esses resultados quantitativos sugerem a satisfação dos participantes e a pertinência da proposta base do !F Lab - treinar o desenvolvimento de projetos de narrativas interativas e imersivas baseado em user centre desing - e das experimentações do !F Bug Lab - Strategy Canvas e o incremento de momentos de trocas diretas entre os participantes. Essa sugestão fica mais evidente nas respostas às perguntas abertas, quando perguntados pelas "modificações efetivas trazidas pela oficina" e "as contribuições principais" para projetos futuros, foram apontadas diversas modificações nos projetos e destacada a importância de uma perspectiva de UCD e estratégica que articula as diversas componentes e fases dos projetos. Em relação às insatisfações, por outro lado, quando foram pedidas "sugestões" para aprimoramento da metodologia, oito (66\%) das doze 
pessoas destacaram a necessidade da oficina ser mais longa e ter mais tempos para o desenvolvimento das atividades.

\section{CONSIDERAÇÕES}

Este artigo descreve o processo de pesquisa-ação da concepção, implementação e experimentação reiterativas do programa de treinamento !F Lab e sua metodologia WHAT IF IT entre 2015 e 2018, em especial o recorte sobre as questões que dizem respeito à tensão entre as experiência do usuário e autor e ao papel dos recursos na elaboração da estratégia do projeto e na concepção do produto narrativo. Como pesquisa-ação, esse percurso começa com a criação do programa em 2015 e se estende a do !F Bug Lab em 2018, a partir da observação participante dos autores sobre o cenário das narrativas interativas e imersivas. Com a realização das oficinas, a pesquisa busca sobretudo escutar o usuário da própria metodologia: os participantes dos programas de treinamento.

Os resultados das pesquisas do !F Lab 2017 e !F Bug Lab são rodadas cíclicas dessa escuta que, de certa forma, coloca em questão as pressuposições teóricas dos programas e aponta os efeitos a curto prazo da experiência dos participantes. Eles podem sugerir efeitos a longo prazo, mas está muito além do alcance das entrevistas e questionários inferir se os participantes incorporam realmente os princípios e práticas do programa ou se a concepção estratégica baseada nos recursos aumentou a possibilidade de realização dos projetos. As investigações das entrevistas e questionários desenham as impressões dos participantesusuários e servem para um melhor entendimento de suas experiências, se justificando tanto para o avanço na compreensão da aplicação da metodologia como no aprimoramento da mesma. Trata-se de um processo de sutis aproximações e lapidações de uma prática e não, de grandes surpresas e descobertas. Nesse sentido, a descrição narrativa revela detalhes do percurso não contemplados nos resultados das entrevistas e questionários.

O perfil dos participantes do !F Bug Lab se assemelhou bastante ao correspondente nos primeiros !F Labs - de origem audiovisual. A disponibilidade de recursos era, como esperado, significativamente menor, sobretudo em relação aos apoio e financiamento. Os resultados encontrados dizem respeito a essa amostra concreta de participantes. Nesse sentido, ficou claro a pertinência do programa de treinamento não apenas pela satisfação e avaliação explícita em termos quantitativos e qualitativos, mas em função da amplitude e profundidade 
de transformações pelas quais seus projetos passaram durante apenas quatro dias de oficina em setembro de 2018 no Rio de Janeiro. Se passaram por essas mudanças, é por que não haviam de forma sistemática sido repensados por uma perspectiva estratégica e de UCD.

Os resultados, no entanto, não dizem nada a respeito dos efeitos concretos nos projetos e seus percursos posteriores. Seria interessante uma pesquisa que pudesse acompanhar a longo prazo questões como: o quanto os participantes incorporam as práticas e princípios na elaboração outros projetos; em que sentido a metodologia repercute na produção e realização dos projetos esboçados nas oficinas; quais mudanças ocorridas nos projetos durante as oficinas são realizadas posteriormente. Esse talvez seja o propósito de uma pesquisa futura por parte dos autores deste artigo. E, quem sabe, os resultados possam ser utilizados para mais uma rodada reiterativa de aprimoramento da metodologia WHAT IF IT. Nesse sentido, a capacidade de mobilização de recursos para a produção de novos treinamentos será um ponto chave.

\section{REFERÊNCIAS}

ASTON, J.; GAUDENZI S. Interactive Documentary: Setting the Field. Studies in Documentary Film, (6)2, 125-139, 2012.

ASTON, J.; GAUDENZI, S.; ROSE, M. I-Docs: The Envolving Practices of Interactive Documentary. London and New York: Columbia University Press, 2017.

BOLE, N.; CÉDRIC, M. Le webdoc existe-t-il? Paris: Le Blog Documentaire éditions, 2014.

DESROCHE, H. Pesquisa-ação: dos projetos de autores aos projetos de atores e vice-versa. In. THIOLLENT, M. (org.). Pesquisa-ação e projeto cooperativo na perspectiva de Henri Desroche.. São Carlos: EdUFSCar, pp. 33-68, 2006.

GAUDENZI, S. The Interactive Documentary as a Living Documentary. Revista Doc On Line, 14, p. 9-31, 2013b. Acesso em 30 de Agosto de 2017. http://www.doc.ubi.pt/

GAUDENZI, S. The Living Documentary: from representing reality to co-creating reality in digital interactive documentary. Thesis. London: University of London, 2013a.

GAUDENZI, S. User experience versus author experience: lesson learned from the UX Series. In. ASTON, J.; GAUDENZI, S.; ROSE, M. In. I-Docs: The Envolving Practices of Interactive Documentary. London and New York: Columbia University Press, 2017 (Kindle).

GAUDENZI, S; WEILER, L. The Learn Do Share design methodology: Lance Weiler in conversation. In. ASTON, J.; GAUDENZI, S.; ROSE, M. In. I-Docs: The Envolving Practices of Interactive Documentary. London and New York: Columbia University Press, 2017 (Kindle). 
GIFREU, A. El Documental interactivo en la estrategia de la multidifusión digital: evaluación del estado del arte en relación con la temática, las plataformas y la experiencia del usuário.

Revista TELOS (Cuadernos de Comunicación e Innovación) pp. 1-11, 2014.

GIFREU, A. EI Documental Interactivo: evolución, caracterización y perspectivas de desarrollo. Barcelona: UOCPress, 2013.

JENKINS, H. Convergence Culture: Where Old and New Media Collide. New York: NYU Press, 2006.

JENKINS, H..; FORD, S; GREEN, J. Spredable media: Creating Value and Meaning in a Networked Culture. New York: NYU Press, 2013.

LANDOW, G. Hypertext 3.0: critical theory and new media in an era of globalization. Baltimore: Johns Hopkins, 2006.

LININGTON, J. Pushing the craft forward: the POV Hackathon as a collaborative approach to making an interactive documentary. In. I-Docs: The Envolving Practices of Interactive Documentary. London and New York: Columbia University Press, 2017 (Kindle).

MURRAY, J. Hamlet no holodeck: o futuro da narrativa no ciberespaço. São Paulo: Itaú Cultural; UNESP, 2003.

NASH, K. Modes of interactivity: analysing the webdoc. Media, Culture \& Society, 34 (2), pp. 195-210, 2012.

NASH, K; CORNER, J. Strategic impact documentary: Contexts of production and social intervention. European Journal of Communication. pp. 1-16, 2016.

$\mathrm{NASH}, \mathrm{K}$. What is interactivity for? The social dimension of web-documentary participation. Continuum: Journal of Media \& Cultural Studies. pp. 37-41, 2014.

PAZ, A.; SALLES, J. Dispositivo, Acaso e Criatividade: Por uma estética relacional do webdocumentário. Revista Doc On Line 14, pp. 33-69, 2013. Acesso em 30 de Agosto de 2017. http://www.doc.ubi.pt/.

PAZ, A.; SALLES, J. Brasil, Mostra a sua Cara: aproximações ao cenário brasileiro de documentários interativos. Revista Doc On Line, 18, pp. 130-165, 2015. Acesso em 30 de Agosto de 2017. http://www.doc.ubi.pt/.

PAZ, A. Documentário interativo e estratégia de impacto social. Revista Doc On Line Num. Esp., pp. 81-108, 2017. Acesso em 30 de Agosto de 2017. http://www.doc.ubi.pt/.

PAZ, A.; GAUDENZI, S. (Orgs). Bug: narrativas interativas e imersivas. Rio de Janeiro: Automática, Letra e Imagem e Oi (Coedição), 2019.

PAZ, A.; JUCÁ, M. O Cenário Inovador das Narrativas Interativas e Imersivas. In. PAZ, A.; GAUDENZI, S. (Orgs). Bug: narrativas interativas e imersivas. Rio de Janeiro: Automática, Letra e Imagem e Oi (Coedição), pp. 9-17, 2018. 
PAZ, A.; MACIEL, K. A. Além das Interfaces: conceitos e obras fundamentais de Narrativas Interativas. In. PAZ, A.; GAUDENZI, S. (Orgs). Bug: narrativas interativas e imersivas. Rio de Janeiro: Automática, Letra e Imagem e Oi (Coedição), pp. 44-59, 2018.

PRINGLE, R. Testing and evaluating design prototypes: the case study of Avatar Secrets. In. I-Docs: The Envolving Practices of Interactive Documentary. London and New York:

Columbia University Press, 2017 (Kindle).

SARASVHATHY, S. ; DEW, N.. New Market creation through transformation. Journal of Evolutionary Economics, 15, pp. 533-565, 2005.

SARASVHATHY, S.. Causation and Effectuation: Toward a theoretical shift from economic inevitability to entrepreneurial contingency. Academy of Management Review, 262, pp. 243-288, 2001.

SARASVHATHY, S. Entrepreneurship as a Science of the artificial. Journal of Economic Psychology 24, pp. 203-22, 2003.

SCOLARI, C. Narrativas transmedia. Cuando todos los medios cuentan. Barcelona: Deusto, 2013.

Original recebido em: 29 de março de 2019

Aceito para publicação em: 01 de setembro de 2019

André Paz

Professor, pesquisador e curador focado no campo das narrativas interativas e imersivas. É professor adjunto da Universidade Federal do Estado do Rio de Janeiro (UNIRIO). Professor credenciado no Mestrado Profissional em Criação e Produção de Conteúdos Digitais, do Programa de Pós-Graduação em Tecnologias e Linguagens da Comunicação (PPGTLCOM) da Universidade Federal do Rio de Janeiro (UFRJ). Nos últimos anos trabalha com pesquisaação de metodologias de desenvolvimento de projetos. Coordena na UNIRIO o Programa de

Extensão Buglab III: tecnologia, produção e imagem. É coordenador e editor do Bug404 (bug404.net), rede e portal que dissemina e apoia a inovação em narrativas interativas e imersivas. Curador na Mostra Bug.

\section{Sandra Gaudenzi}

Professora, pesquisadora e consultora do campo das narrativas interativas e imersivas. Organizadora e autora do livro The Envolving Practices of Interactive Documentary (2017). Idealizadora e Professora do programa de mestrado disLAB (Digital and Interactive Storytelling $L A B M A$ ), da University of Westminster, Londres. Idealizadora, Pesquisadora e Curadora do grupo de pesquisa e conferência i-Docs (i-docs.org), do Digital and Cultural Research Center da University of West of England.

Idealizadora e coordenadora do !F Lab, Interactive Factual Lab, programa de treinamento financiado pela Creative Europe da União Européia (iflab.eu). 


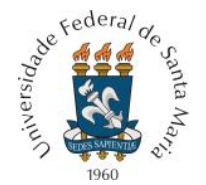

PROGRAMA DE PÓS-GRADUAÇÃO EM COMUNICAÇÃO DA UNIVERSIDADE FEDERAL DE SANTA MARIA

\section{(-) (О)(-}

Esta obra está licenciado com uma Licença

Creative Commons Atribuição-NãoComercial-CompartilhaIgual 4.0 Internacional 